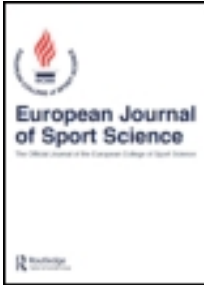

\title{
Especial skill vs. Quiet Eye duration in basketball free throw: Evidence for the inhibition of competing task solutions
}

\begin{tabular}{|r|l|}
\hline Journal: & European Journal of Sports Science \\
\hline Manuscript ID & TEJS-2018-0522.R2 \\
\hline Manuscript Type: & Original Paper \\
\hline Keywords: & Skill, Motor Control, Team Sport, Theory \\
\hline \multicolumn{2}{|l}{} \\
\end{tabular}

\section{SCHOLARONE \\ Manuscripts}




\begin{abstract}
The quiet eye $(\mathrm{QE})$ is a gaze phenomenon that has been studied over more than two decades. However, the underlying mechanisms of the well-known expertise effect, viz, longer QE durations in experts when compared to less-skilled athletes remain unclear. Therefore, from a functional perspective an inhibition hypothesis was proposed that explains long QE durations in experts with increased inhibition requirements over movement parametrization. This hypothesis was tested by making use of the especial-skill effect in basketball free throw which refers to the observation of higher actual performance than would be predicted on the basis of performance at the nearby locations. In line with the expectations, from the distance of the free-throw line, higher actual than predicted shooting accuracy and longer actual than predicted QE duration, were revealed. This suggests that when performing free throws prolonged QE durations are required to shield the optimal against alternative task solutions within the very dense sub-space of this especial skill. These findings suggests an inhibition function of long QE durations in expert athletes.
\end{abstract}

Key words: Perception-action coupling, gaze behavior, expertise, motor control, sports 
The study on the mutual coupling between perception and action has gained considerable attention over the last decades and it has been shown that superior performance in cognitive and motor skills comes along with distinct differences in perceptual-cognitive skills (for recent meta analyses see Gegenfurtner, Lehtinen, \& Säljö, 2011; Mann, Williams, Ward, \& Janelle, 2007). In a nutshell, evidence suggests that experts show 'optimal' gaze behavior which allows to perceive crucial information synchronized to the execution of a motor action. For example, in sports as darts or basketball, which require to throw an object as precise as possible at a target in space, it has been shown that the duration of stable gaze behavior (i.e., fixations) at the target to be hit, explains a significant amount of variance within motor expertise and motor performance. Vickers (1996) was the first to describe this gaze phenomenon and since then the functionality of the Quiet Eye (QE) has been replicated for a large number of different sport tasks (for recent overview, see Vickers, 2016). The QE is defined as the final fixation at an object in space just before the initiation of the crucial phase of the movement. For example, in basketball free throw - with the rim of the basket as the object in space and the final extension of the throwing arm just before the throwing action as the crucial phase of the movement (Vickers, 1996) - experts showed twice as long QE durations when compared to their less-skilled counterparts (about $900 \mathrm{~ms}$ vs. $400 \mathrm{~ms}$ ). Moreover, differences in QE duration were also observed when comparing successful vs. unsuccessful shooting attempts with longer QE durations for the former (e.g., Vickers, 1996). Recently, these two effects were summarized in a meta-analysis by Lebeau et al. (2016) who found a large effect size $(d=1.04)$ for the QE-expertise effect and a medium effect size $(d=$ 0.58) for the QE-performance effect.

Regarding potential mechanisms, the QE phenomenon has been explained with improved information processing over movement parametrization. Vickers (1996, p. 351; see also Gonzales et al., 2015) suggested that the QE subserves "to adequately set the parameters 
of the shot, such as the location and distance to the target, the trajectory on the ball, the optimal forces needed throughout the action, the timing, and the coordination of the limb". Among others, this optimized-movement-parametrization hypothesis was tested by Williams, Singer, and Frehlich (2002) who manipulated task demands in a billiard task. As predicted, increased shot difficulty resulted in increased QE durations owing to increased information processing in movement preparation (on the relation between task demands and information processing, e.g., Klapp, 1975). Since then, this relation has been replicated and extended. For example, in a golf-putting task Walter-Symons, Wilson, Klostermann, and Vine (2018) found increased QE duration with increasing task difficulty (i.e., distance to the target, size of the hole, and size of the putter surface). Moreover, Klostermann, Kredel, and Hossner (2013) showed that also the performance-enhancing effect of long QE durations is attributable to information-processing demands. In their studies, experimentally controlled long QE durations improved throwing accuracy in a targeting task only if the position of a target to be hit was not predictable, thus, had to be processed over the QE duration (for further studies that have provided evidence in support of the optimized-movement-parametrization hypothesis see also Horn, Okumura, Alexander, Gardin, \& Sylvester,2012; Mann, Coombes, Mousseau, \& Janelle, 2011; Moore, Vine, Cooke, Ring, \& Wilson, 2012).

Moreover, Vine, Moore, and Wilson (2014) suggested improved attentional control processes to the explanation of the QE phenomenon. In several learning studies (e.g., Vine \& Wilson, 2010; Vine \& Wilson, 2011; Vine, Moore, \& Wilson, 2011) Vine and colleagues showed that participants trained with QE interventions in a golf-putting and basketball-freethrow task not only outperformed active control groups in posttest and retention tests. But also, in situations with experimentally evoked increased pressure, the QE-intervention groups maintained performance whereas the active control groups showed a clear drop in putting and shooting accuracy, respectively. In light of these findings, Vine et al. (2014, p 238) proposed 
that "the QE helps to maintain effective goal-driven attentional control, while reducing the impact of the stimulus-driven attentional system" (on the effect of pressure on attentional control, e.g., Eysenck, Derakshan, Santos, \& Calvo, 2007). Thus, over the QE period sufficient amount of cognitive resources can be allocated for ongoing movement parametrization (Vine et al., 2011).

Both mechanisms provide a sound explanation for the QE-performance relation in 'regular' situations (e.g., Williams et al., 2002) as well as in pressurized situations (e.g., Vine \& Wilson, 2010). But, they hardly can explain why experts show longer QE durations when compared to their less-skilled counterparts because increased information-processing demands and increased attentional load, respectively, in experts must be assumed. However, by knowing that motor control processes become automatized over motor learning (e.g., Schmidt \& Lee, 2011), this assumption seems unlikely (see also Mann, Wright, \& Janelle, 2016). Therefore, Klostermann, Kredel, and Hossner (2014) proposed a development of the existing mechanisms by introducing the inhibition hypothesis. Drawing on a functional framework (e.g., Allport, 1987; Neumann, 1996), the inhibition hypothesis suggests that the QE is used as shielding mechanism which promotes the parametrization of the optimal task solution. Thus, further solutions for the given task must be inhibited to prevent interferences over movement parametrization (see also Cisek \& Kalaska, 2010). In experts, the demand to inhibit alternative task solutions can be assumed to be larger as for less skilled athletes as they should have experienced a larger number of very similar task solutions over motor learning. Consequently, this difference in inhibition demands should be reflected in different QE durations with experts requiring longer QE durations than the less skilled athletes. Hence, based on this hypothetical mechanism, the inhibition hypothesis might offer an explanation for the QE expertise effect. 
Recently, Klostermann and Hossner (2018) tested this hypothetical mechanism in a perceptual-learning study in which participants practiced a throwing task with a high-extend vs. a low-extend training protocol. The high-extend training required to throw balls at a large number of positions spaced regularly at the vertical axis of a screen presented in front of the participants. In contrast, the low-extend protocol required to throw at two different positions only, one in the upper and one in the lower half of the same vertical axis. Thus, the two protocols differed in the extension of the task solution space to be learned. The high-extend learning group experienced a high number of task variants over one single task-solution space with its boundaries at the most outward target positions at the top and at the bottom. In contrast, the low-extend learning group experienced a low number of task variants distributed over two separate sub spaces. It was predicted that the high number of task solutions distributed over one task solution space would result in increased inhibition demands and longer QE durations, respectively in posttest and retention test. However, after learning the groups did not significantly differ and rather the low-extend learning group showed longer QE durations. Thus, it was argued that it might not be the extension but the density, i.e. the closeness of movement effects one experiences over motor learning, of the task solution space affecting inhibition demands over movement parametrization.

Such dense task solutions spaces should be particularly present in motor skills that have been trained with very similar task solutions under standardized conditions. Recent research suggests that the free-throw in basketball could correspond to these density requirements, in particular, in differentiation to other set shots in basketball. In this regard, Keetch, Schmidt, Lee, and Young (2005) showed that experts taking shots from the distance of the free-throw line performed significantly better than would be predicted by the relationship of the accuracies of set shots attempted at different distances. This phenomenon was labelled "especial skill” (for an overview, e.g., Keetch, Lee, \& Schmidt, 2008). Drawing 
on Schmidt's schema theory (1975), it is suggested that the free-throw represents a highly specific capability within the general class of set shots which results from the extensive practice of this specific skill (Keetch et al., 2008). Therefore, it can be assumed that high amount of practice establishes a single and very dense sub-space for the free throw within the task-solution space of the set shot. Consequently, the especial-skill approach might be a perfect experimental tool to study the assumed relation between the density of a task-solution space, inhibition demands, and the QE duration.

Therefore, in the current study the effects of an especial skill in basketball on basketball players' motor performance and QE duration was investigated. To this end, basketball players had to shoot from five different distances with two of them being either closer or farther away from the free-throw line. As a manipulation check, it was tested whether the basketball players would perform better from the free-throw distance as predicted based on the relation between shooting distance and shooting accuracy (e.g., Keetch et al., 2005). More crucially, it was expected that the relation between QE duration and distance (e.g. Walter-Symons et al., 2017) also would be broken at the distance from the free-throw line. Based on the inhibition hypothesis, it was expected that the actual QE duration would be longer than the predicted QE duration.

\author{
Method \\ Participants \\ Participants were 15 male and one female basketball players $(M$ age $=23.3$ years, $S D$ \\ age $=6.4$ years) recruited from sport science students and local basketball clubs. Due to the \\ novelty of the research question, the calculation of the sample size was based on the sample in \\ earlier studies on the especial skill effect (e.g., $n=8$ Keetch et al., 2005; $n=12$ Stöckel \&
}


Breslin, 2013) and the QE (e.g., $n=8$ experts in Vickers, 1996). The players had on average 10.4 years $(S D=5.2$ years) basketball experience and trained for 9.9 years $(S D=5.2$ years $)$. The self-reported average seasonal statistics were $67.0 \%(S D=9.5 \%)$ for free throws, 48.3 $\%(S D=16.0 \%)$ for field goals, and $35.2 \%(S D=10.4 \%)$ for 3 -point shots. Since one male participant could not finish data collection due to technical problems, the player had to be removed from the sample. Written informed consent from the participants was obtained in advance. The study was carried out in accordance with the 1964 Declaration of Helsinki. The protocol was approved by the ethics committee of the local Faculty of Human Sciences.

\section{Apparatus}

All trials were recorded with a GoPro camera (Hero 4 black, GoPro Inc., San Mateo, USA, $30 \mathrm{~Hz}$ ) that was positioned perpendicular to the free-throw line at a distance of $5 \mathrm{~m}$. The participants' eye movements were recorded with a Pupil mobile eye tracker (Pupil Labs GmbH, Berlin, Germany, world camera: $30 \mathrm{~Hz}$; eye camera: $200 \mathrm{~Hz}$ ) (see also Kassner, Patera, \& Bulling, 2014) which was connected to a MacBook Air (Apple Co., Cupertino, USA) via a 10 m long USB active optical cable (Unibrain, San Ramon, USA) with the power supply stored in a light rucksack. Prior to testing, the eye tracker was calibrated using a 5point calibration grid presented at a distance of $4.23 \mathrm{~m}$. The calibration was checked after each series of 6 shots and the system was re-calibrated if necessary. In the beginning of each block of 30 trials, a small light was flashed for later synchronization of the recordings from the GoPro camera and the Pupil mobile eye tracker.

Experimental setup 
The study was conducted at a regular training facility of the Sport Science Institute with an official FIBA regulation court. The shots were taken with an official basketball (Spalding Co., Bowling Green, USA, Size: 6 for the female and 7 for the male participants) into a regular-sized basketball rim mounted at a height of $3.05 \mathrm{~m}$. Like in earlier studies (e.g., Stöckel \& Breslin, 2013), the shots were taken from five different distances facing the front on to the basket: $3.63 \mathrm{~m}, 3.93 \mathrm{~m}, 4.23 \mathrm{~m}$ (i.e., the free throw line according to the official FIBA rules), $4.53 \mathrm{~m}$, and $4.83 \mathrm{~m}$. The five shooting positions were marked using masking tape. One experimenter and the recording devices were positioned to the left of the GoPro camera. The second experimenter was positioned next to the basket returning the balls after each shot.

Procedure

Each participant attended individual sessions. After an individual warm-up, the participants were equipped with the Pupil mobile eye tracker and additional 20 warm-up shots from all five distances were conducted to accommodate to the slightly different testing situation. In the following, the Pupil mobile eye tracker was calibrated and the testing started with the first out of five blocks. In each block, the participants performed 6 shots from each of the five shooting distances. Thus, a total of 150 shots were taken with 30 shots from each shooting distance. For each block, the 6 shots per distance were performed in succession with the order of distances randomized (cf. Stöckel \& Breslin, 2013). The participants performed the shots at their own pace. At the end of the testing, the participants were thanked and debriefed about the aims of the study. Each session lasted about 90 minutes.

Data analysis 
For each of the five shooting distances, shooting accuracy was determined by summing the number of successful attempts and dividing it by the number of test trials. To obtain percentage scores this value was multiplied by 100 . To test for the especial skill effect, for each participant linear regressions were calculated on the basis of the distances $3.63 \mathrm{~m}$, $3.93 \mathrm{~m}, 4.53 \mathrm{~m}$, and $4.83 \mathrm{~m}$. With the individual slope and intercept values, the predicted value for the free-throw distance (i.e., $4.23 \mathrm{~m}$ ) was calculated and compared to the actual value by means of a dependent t-test (see Keetch et al., 2005).

For the gaze analyses, the recordings of the Pupil eye tracker and the GoPro camera were synchronized using the Kinovea 0.8 .15 video chronometer and motion-analysis software (Boston, MA, USA). In more detail, in both recordings the time stamp the frame of the light flash was recorded. In case that the light flash was not seen by both video cameras $(n=3,3.8$ $\%$ of all cases), the frame of the ball touching the basket was taken as specific event. Subsequently, for the QE calculation the time stamp of the initiation of the critical movement phase was extracted from the GoPro recordings - i.e., the frame of the final extension of the shooting arm prior to ball release (cf. Vine \& Wilson, 2011; see also Wilson, Vine, \& Wood, 2009).

The gaze data were analyzed with a semi-automatic procedure using a self-written Matlab script (Mathworks, Natick, USA) and the Pupil Player software (Pupil Labs GmbH, Berlin, Germany). First, from the recordings of the Pupil mobile eye tracker the raw gaze data and the video recordings from the world camera were exported. From the raw gaze data, the relative horizontal and vertical pixel positions of the gaze cursor in the reference frame of the world camera were taken to calculate fixations with a dispersion-based algorithm (e.g., Nyström \& Holmqvist, 2010): A fixation was detected if the relative pixel positions remained stable within $3^{\circ}$ of visual angle for at least $120 \mathrm{~ms}$, (e.g., Vickers, 1996). The $3^{\circ}$-visual-angle threshold was estimated separately for each of the five distances by (1) calculating the visual 
angle of the upper frame of the basket as a function of the throwing distances (i.e., the arc tangent of the size of the upper frame of the basket divided by the distance to the basket), (2) recalculating the visual angle into pixels (i.e., dividing the visual angle by the size of the upper frame of the board in pixels), and (3) recalculating the visual angle per pixels into visual angle per relative pixels (i.e., dividing the absolute value by the resolution of the video of the world camera). At this stage, for each participant fixations over the 5 different recordings (containing 30 trials each) were calculated.

In the following, for each trial the last fixation before the initiation of the critical movement phase was extracted and the corresponding picture was read from the video recordings which displayed the position of the gaze cursor in the world camera in the final fixation period, thus the position of the QE. In the last step, all pictures were controlled manually by one naïve experimenter and all trials were removed if the gaze cursor was not positioned at the basket (cf. Vickers, 1996). Summarizing, fixations were calculated by use of the raw gaze data applying a dispersion-based algorithm. In the following, the final fixation before the initiation and the corresponding video picture were extracted. Finally, the gaze position in the pictures were manually controlled and the trials in which the gaze was not positioned at the basket were discarded from further analyses.

The first check of the gaze data revealed that no QE was found for on average in $4.2 \%$ of all trials (min: $0 \%$, max: $29.3 \%$ ) because of missing fixation detection before movement initiation and for on average $8.2 \%$ of all trials (min: $0 \%$, max: $19.3 \%$ ) because of inaccurate gaze position within the final fixation. In sum, for $12.4 \%(S D=13.5 \%)$ of all data no $\mathrm{QE}$ could be calculated. When considering the quality of the gaze data individually, it was apparent that in particular two participants had insufficient QE data (40.6\% and $46.0 \%$ of all trials without QE detection). Therefore, the gaze data of these two participants were removed from further analyses such that the gaze data of 13 participants were available with on average 
137.3 gaze-data trials. The number of gaze-data trials differed only slightly between the five distance conditions with on average the lowest amount for the $3.93 \mathrm{~m}$ condition $(\mathrm{M}=26.7$ trials) and the highest amount in the $3.73 \mathrm{~m}$ and the 5.23 conditions $(\mathrm{M}=27.9$ trials $)$. The split-half reliability of the QE detection proved to be excellent: Spearman-Brown-Coefficient $r=.83$.

Also for QE duration, individual linear regressions were calculated for the different shooting positions apart from the $4.23 \mathrm{~m}$ distance. Subsequently, for each participant the predicted value was calculated and compared to the empirical value by means of a dependent $t$ test.

In addition, to assess the QE-performance relationship, for each participant a median split of the QE duration was applied to separate trials with long vs. short QE durations. This resulted in a successful median split with significantly longer QE durations in long $(M=$ 1003.4 ms, $S D=413.5 \mathrm{~ms})$ when compared to short $(M=358.1 \mathrm{~ms}, S D=182.8 \mathrm{~ms}) \mathrm{QE}-$ duration trials, $t(12)=8.13, p=.000, d=2.25$. In the following, shooting accuracy was separately aggregated for long vs. short QE-duration trials and tested on differences with a dependent $t$ test.

For all inferential tests, the significance level was set to $\alpha=.05$. The effect sizes were computed as Cohen's $d$-value.

\section{Results}

In Figure 1, shooting accuracy for each of the five shooting distances as well as the predicted shooting accuracy at $4.23 \mathrm{~m}$ is displayed. As revealed by a linear regression, the shooting accuracy decreased with increasing distance which, on average, accounted for 64.2 $\%$ of the variance in the data. On the basis of the individual regression equations, the 
predicted shooting accuracy at the distance of $4.23 \mathrm{~m}$ was $66.7 \%(S D=11.8 \%)$. However, the basketball players' actual performance at this distance was $73.1 \%(S D=12.2 \%)$. The difference between predicted and actual performance was significant, $t(14)=4.35, p=.001, d$ $=1.12,95 \% \mathrm{CI}[3.2,9.5]$, replicating the especial skill effect.

$<<<$ Please insert Figure 1 about here $>>>$

For QE duration (Figure 2), an increase in QE duration as a function of shooting distance was found $\left(\mathrm{r}^{2}=.50\right)$. With regards to the crucial comparison of the predicted and the actual QE duration at the free throw distance (i.e., $4.23 \mathrm{~m}$ ), participants actual QE durations $(M=764.7 \mathrm{~ms}, S D=352.0 \mathrm{~ms})$ were longer as the predicted QE durations $(M=658.5 \mathrm{~ms}, S D$ $=297.1 \mathrm{~ms}), t(12)=3.42, \mathrm{p}=.001, d=0.95,95 \% \mathrm{CI}[38.6,173.9]^{1}$.

$<<<$ Please insert Figure 2 about here $>>>$

When comparing shooting accuracy as a function of QE duration, participants were slightly more accurate in long $(M=69.7 \%, S D=11.2 \%)$ vs. short $(M=68.3 \%, S D=13.5$ \%) QE-duration trials. However, this difference clearly failed to reach the pre-determined level of significance, $t(12)=0.59, p=.56, d=0.16,95 \%$ CI $[-3.5,6.2]^{2}$. When considering the QE-performance relation for the free-throw distance only, the pattern of result remained the same with slightly higher shooting accuracies for the long (QE duration: $M=1127.5 \mathrm{~ms}$; shooting accuracy: $M=74.8 \%, S D=12.9 \%$ ) when compared to the short QE-duration trials (QE duration: $M=404.3 \mathrm{~ms}$; shooting accuracy: $M=73.9 \%, S D=12.5 \%$ ), $t(12)=0.25, p=$ 
$.84, d=0.05$. Consequently, intra-individual performance effects of long QE durations could not be revealed.

\section{Discussion}

This study aimed to investigate a hypothesized mechanism of the well-known but underexplored QE expertise effect, i.e., longer QE duration in experts when compared to their less-skilled counterparts. In contrast to other explanations, the inhibition hypothesis might offers an explanation to this phenomenon. According to this mechanism, experts require longer QE durations to inhibit the larger number of experienced task solutions that are competing over movement parametrization. To test this assumption, QE duration and motor performance were investigated in basketball set shots that were taken from different shooting distances. Drawing on the especial skill effect, it was expected to reveal higher actual than predicted shooting accuracy at the distance of the free-throw line. With regards to the QE a similar effect was expected, viz, longer actual than predicted QE durations at the free-throw distance.

The analysis of the performance data revealed a successful manipulation check. The basketball players showed a significantly higher shooting accuracy from the free-throw line than predicted. This finding replicates the especial skill effect in basketball set shots (e.g., Stöckel \& Breslin, 2013) and allows to discuss the QE data with regards to the experimental manipulation.

When referring to the QE data (Figure 2), the expected difference between the predicted QE duration and the actual QE duration at the shooting distance from the free-throw line was evident. The basketball players showed about $20 \%$ longer QE durations than on the basis of the individual regression coefficients would have been predicted. Therefore, this 
finding is in line with the assumed mechanism of the inhibition hypothesis. Due to the high amount of practice from this shooting distance, the free throw has established a single and very dense sub-space within the general task solution space of set shots (see also Keetch et al., 2008). This high density evoked by the very high amount of very similar shot repetitions, required increased inhibition, thus longer QE durations. In addition, this relation between motor expertise and inhibition processes over the QE period could be further corroborated when taking the years of practice into account. However, post-hoc correlational analysis between the years of practice and the size of the QE effect (i.e., the difference between actual and predicted QE duration) revealed only a moderate correlation $\left(r_{\mathrm{s}}=.42, p=.15\right)$. This means that the higher the amount of movement experience the more inhibition over movement parametrization was required. Nonetheless, overlooking the inferential statistics this result should be taken carefully and requires further research. Moreover, the relevance of factors like familiarity with the distance and possible distraction by different visual cues should be addressed in future studies.

When discussing the current results in light of the response-programming approaches (e.g., Vickers, 1996; Vine et al., 2014), the necessity to further advance these mechanisms should become apparent because the exact opposite pattern should have been revealed. As sketched above, ensuing from these approaches the QE is considered as a quantitative and qualitative index of information processing. Having in mind that over motor learning a reduction in processing demands must be expected, in the current study a shorter actual than predicted QE duration should have been found due to the lower parametrization requirements and rather "automatic" control processes, respectively for the set shot from the free-throw distance.

The limitations of the study have to be acknowledged. First, it has to be noted that different to earlier studies (e.g., Stöckel \& Breslin, 2013) the relation between shooting 
accuracy and shooting distance was not so strong as expected $\left(r^{2}=.64\right)$. When referring to Figure 1, it becomes apparent that the shooting accuracy at the shortest distance was surprisingly low and not better than performance from the second distance. Post-hoc analyses were conducted to test whether this effect could be explained by the participants' playing position which, however, was not the case. Thus, on the basis of the current data one hardly can explain this finding. Second, no performance-enhancing effects of long QE durations was revealed. Distinct differences in QE duration did not result in significant differences in throwing accuracy neither over all distances nor over the free-throw distance only. However, as also reported by Lebeau et al. (2016) already in earlier studies researchers failed to show a clear QE-performance relation. In the current case, one could speculate that, e.g., the data analysis applied - i.e. QE median split - might not be valid to study this relation. However, it has to be noted that the current study did not intend to study QE-performance effects such that also the experimental was not designed with regards to this question.

In summary, to the best of my knowledge this is the first study that investigated the relation between the QE and the especial skill effect in basketball set shots. It was shown that, like throwing accuracy, the QE duration breaks the otherwise strong relation between task demands and motor behaviour at the highly trained distance of $4.23 \mathrm{~m}$. This suggests increased inhibition demands due to high amount of movement experience for this specific skill. Nevertheless, future studies need to further investigate this relation. For example, when applying training studies one should look at the development of this two phenomena. In this regard, first approaches can be found for the especial skill effect (Breslin, Hodges, Steenson, $\&$ Williams, 2012). But, the current results suggest the strong interplay between perception and action. Therefore, the full picture will allow to better understand both phenomena. 


\section{Footnotes}

1. It should be noted that also with the full sample size $(n=15)$ the QE effect was found. As post-hoc analyzes revealed, participants showed longer actual QE durations $(M=$ $719.8 \mathrm{~ms}, S D=350.7 \mathrm{~ms}$ ) than it was predicted on the basis between shooting distance and QE duration $(M=638.9 \mathrm{~ms}, S D=280.9 \mathrm{~ms}), t(14)=2.47, p=.013, d=0.64,90 \%$ CIs [23.3, 138.3].

2. Different from earlier studies (e.g., Vickers, 1996), QE duration was not calculated as a function of hits vs. misses because of the unequal distribution of hits vs. misses in the current study. However, the pattern of result does not change when calculating QE duration for hits vs. misses. 


\title{
References
}

\author{
Allport, A. (1987). Selection for action: Some behavioral and neurophysiological \\ considerations of attention and action. In H. Heuer \& A. F. Sanders (Eds.), \\ Perspectives on perception and action (pp. 395-419). Hillsdale, NJ: Lawrence \\ Erlbaum.
}

Breslin, G., Hodges, N. J., Steenson, A., \& Williams, A. M. (2012). Constant or variable practice: Recreating the especial skill effect. Acta Psychologica, 140, 154-157.

Breslin, G., Hodges, N. J., Kennedy, R., Hanlon, M., \& Williams, A. M. (2010). An especial skill: Support for a learned parameters hypothesis. Acta Psychologica, 134, 55-60.

Cisek, P., \& Kalaska, J. F. (2005). Neural correlates of reaching decisions in dorsal premotor cortex: specification of multiple direction choices and final selection of action. Neuron, 45, 801-814.

Cisek, P., \& Kalaska, J. F. (2010). Neural mechanisms for interacting with a world full of action choices. Annual Review of Neuroscience, 33, 269-298.

Czyż, S. H., Kwon, O. S., Marzec, J., Styrkowiec, P., \& Breslin, G. (2015). Visual uncertainty influences the extent of an especial skill. Human Movement Science, 44, 143-149.

Eysenck, M. W., Derakhshan, N., Santos, R., \& Calvo, M. G. (2007). Anxiety and cognitive performance: attentional control theory. Emotion, 7, 336-353.

Gegenfurtner, A., Lehtinen, E., \& Säljö, R. (2011). Expertise differences in the comprehension of visualizations: A meta-analysis of eye-tracking research in professional domains. Educational Psychology Review, 23(4), 523-552.

Gonzalez, C. C., Causer, J., Miall, R. C., Grey, M. J., Humphreys, G., \& Williams, A. M. (2015). Identifying the causal mechanisms of the quiet eye. European Journal of Sport Science, 17, 74-84. 
Horn, R. R., Okumura, M. S., Alexander, M. G., Gardin, F. A., \& Sylvester, C. T. (2012). Quiet eye duration is responsive to variability of practice and to the axis of target changes. Research Quarterly for Exercise and Sport, 83, 204-211.

Kassner, M., Patera, W., \& Bulling, A. (2014, September). Pupil: an open source platform for pervasive eye tracking and mobile gaze-based interaction. In Proceedings of the 2014 ACM international joint conference on pervasive and ubiquitous computing: Adjunct publication (pp. 1151-1160). ACM.

Keetch, K. M., Lee, T. D., \& Schmidt, R. A. (2008). Especial skills: Specificity embedded within generality. Journal of Sport \& Exercise Psychology, 30, 723-736.

Keetch, K. M., Schmidt, R. A., Lee, T. D., \& Young, D. E. (2005). Especial Skills: Their emergence with massive amounts of practice. Journal of Experimental Psychology: Human Perception and Performance, 31, 970-978.

Klapp, S. T. (1975). Feedback versus motor programming in the control of aimed movements. Journal of Experimental Psychology: Human Perception and Performance, 104, 14753.

Klostermann, A., \& Hossner, E.-J. (2018). The Quiet Eye and motor expertise: Explaining the “efficiency paradox". Frontiers in Psychology, 9:104. doi: 10.3389/fpsyg.2018.00104

Klostermann, A., Kredel, R., \& Hossner, E.-J. (2013). The "Quiet Eye” and motor performance: Task demands matter! Journal of Experimental Psychology: Human Perception \& Performance, 39, 1270-1278.

Klostermann, A., Kredel, R., \& Hossner, E.-J. (2014). On the interaction of attentional focus and gaze: The quiet eye inhibits focus-related performance decrements. Journal of Sport \& Exercise Psychology, 36, 392-400. 
Mann, D. T., Coombes, S. A., Mousseau, M. B., \& Janelle, C. M. (2011). Quiet eye and the Bereitschaftspotential: Visuomotor mechanisms of expert motor performance. Cognitive Processing, 12, 223-234.

Mann, D. T., Williams, A. M., Ward, P., \& Janelle, C. M. (2007). Perceptual-cognitive expertise in sport: A meta-analysis. Journal of Sport \& Exercise Psychology, 29(4), 457-478.

Mann, D. T. Y., Wright, A., \& Janelle, C. M. (2016). Quiet Eye: The efficiency paradox comment on Vickers. Current Issues in Sport Science, 1:111. doi: 10.15203/CISS_2016.111

Moore, L. J., Vine, S. J., Cooke, A., Ring, C., \& Wilson, M. R. (2012). Quiet eye training expedites motor learning and aids performance under heightened anxiety: The roles of response programming and external attention. Psychophysiology, 49, 1005-1015.

Neumann, O. (1996). Theories of attention. In O. Neumann \& F. Sanders (eds.), Handbook of perception and action (pp. 389-446). San Diego: Academic Press.

Nyström, M., \& Holmqvist, K. (2010). An adaptive algorithm for fixation, saccade, and glissade detection in eyetracking data. Behavior Research Methods, 42, 188-204.

Schmidt, R. A. (1975). A schema theory of discrete motor skill learning. Psychological Review, 82, 225-260.

Schmidt, R. A., \& Lee, T. D. (1999). Motor control and learning: a behavioral approach. Human Kinetics, IL: Champaign.

Stöckel, T., \& Breslin, G. (2013). The influence of visual contextual information on the emergence of the especial skill in basketball. Journal of Sport \& Exercise Psychology, $35(5), 536-541$.

Vickers, J. N. (1996). Visual control when aiming at a far target. Journal of experimental psychology. Human perception and performance, 22, 342-354. 
Vickers, J. N. (2016). Origins and current issues in Quiet Eye research. Current Issues in Sport Science, 1:101. doi: 10.15203/CISS_2016.101

Vine, S. J., Moore, L. J., \& Wilson, M. R. (2011). Quiet eye training facilitates competitive putting performance in elite golfers. Frontiers in psychology, 2:8. doi: 10.3389/fpsyg.2011.00008

Vine, S. J., Moore, L. J., \& Wilson, M. R. (2014). Quiet eye training: The acquisition, refinement and resilient performance of targeting skills. European Journal of Sport Science, 14, 235-242.

Vine, S. J., \& Wilson, M. R. (2010). Quiet eye training: Effects on learning and performance under pressure. Journal of Applied Sport Psychology, 22, 361-376.

Vine, S. J., \& Wilson, M. R. (2011). The influence of quiet eye training and pressure on attention and visuo-motor control. Acta Psychologica, 136, 340-346.

Walters-Symons, R., Wilson, M., Klostermann, A., \& Vine, S. (2018). Examining the response programming function of the Quiet Eye: Do tougher shots need a quieter eye?. Cognitive Processing, 19, 47-52.

Williams, A. M., Singer, R. N., \& Frehlich, S. G. (2002). Quiet eye duration, expertise, and task complexity in near and far aiming tasks. Journal of Motor Behavior, 34, 197-207.

Wilson, M. R., Vine, S. J., \& Wood, G. (2009). The influence of anxiety on visual attentional control in basketball free throw shooting. Journal of Sport \& Exercise Psychology, 31, $152-168$. 
Figure 1. Shooting accuracy (\% hits) as a function of the five shooting distances (from $3.63 \mathrm{~m} \mathrm{until} 4.83 \mathrm{~m}$ ) whereas 4.23 represents the free throw distance. The actual data are presented in filled squares whilst the predicted value at the free throw distance is depicted in the open square. The regression coefficients and the explained variance of the model are depicted in the lower left corner. As can be seen, the predicted value is significantly lower than the actual value.

$338 \times 190 \mathrm{~mm}(300 \times 300$ DPI $)$ 


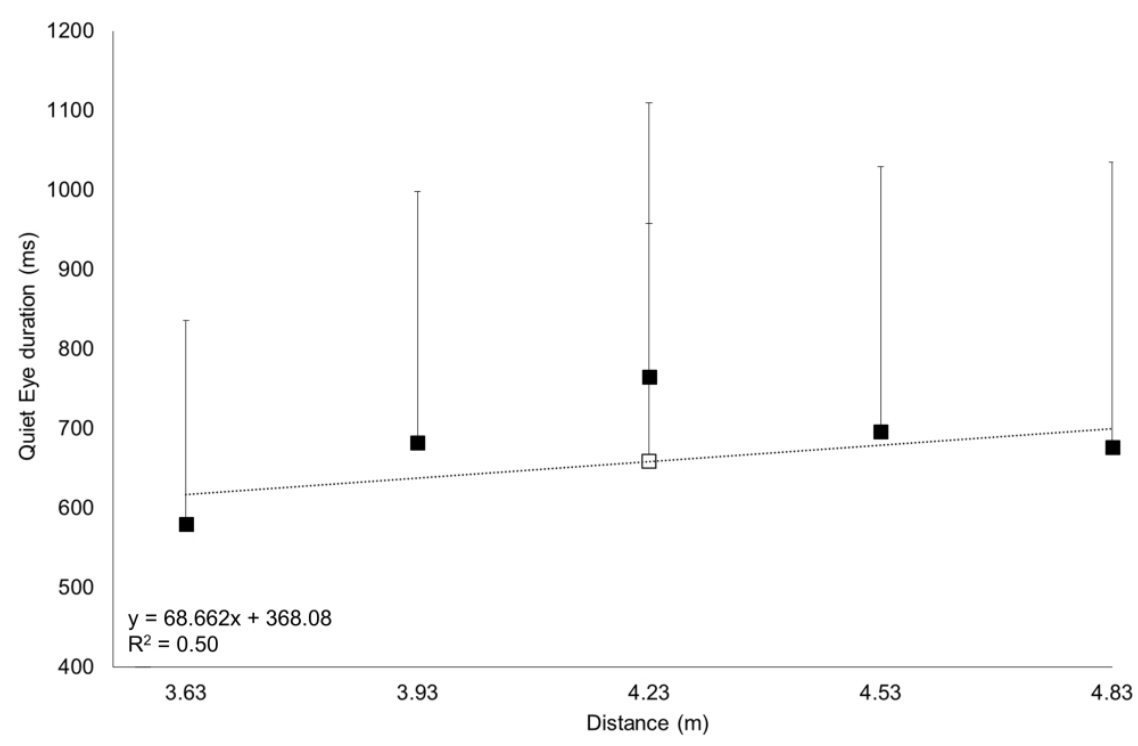

Figure 2. Quiet Eye duration (ms) as a function of the five shooting distances (from $3.63 \mathrm{~m} \mathrm{until} 4.83 \mathrm{~m}$ ) whereas 4.23 represents the free throw distance. The actual data are presented in filled squares whilst the predicted value at the free throw distance is depicted in the open square. The regression coefficients and the explained variance of the model are depicted in the lower left corner. The actual QE duration is significantly longer than the predicted QE duration.

$338 \times 190 \mathrm{~mm}(300 \times 300 \mathrm{DPI})$ 